\title{
ADAPTIVE CARTESIAN MESH REFINEMENT FOR SIMULATING MULTIPHASE FLOWS IN POROUS MEDIA
}

\author{
B. N. CHETVERUSHKIN ${ }^{1}$, N. G. CHURBANOVA ${ }^{1}$, M. A. TRAPEZNIKOVA ${ }^{1}$ \\ A. A. SUKHINOV ${ }^{2}$, AND A. A. MALINOVSKIJ ${ }^{3}$
}

\begin{abstract}
This paper considers two-dimensional hierarchical locally-refined rectangular meshes with dynamic adaptation to the solution. A parallel algorithm with dynamic load balancing has been developed. Adaptive meshes have been used for the problem of passive contaminant transport in an oil-bearing stratum at water flooding. A model neglecting the capillary and gravity forces (the Buckley - Leverett model) has been used.
\end{abstract}

2000 Mathematics Subject Classification: 65M50, 76S05.

Keywords: adaptive mesh refinement, load balancing, interpolation, flow in porous media.

\section{Introduction}

The numerical solution of scientific problems usually requires a grid that covers the computational domain. Discretization of the model equations is performed at the grid element level. Physical phenomena often feature discontinuities and localized high-gradient areas. The numerical simulation of such problems and the conventional techniques (finite elements, finite volumes or spectral methods) with a uniform grid are inefficient when a high accuracy is required. The solution accuracy can be increased through the use of a higher order discretization, or by decreasing the grid elements sizes. The first method has the disadvantage that higher order schemes are prone to product oscillations. Decreasing cell sizes throughout the domain is too expensive.

Adaptive mesh refinement (AMR) is a technique that allows a local refinement of the grid. Thus, adaptivity of the mesh is necessary to cluster grid elements in the regions where it is most needed, while keeping the grid coarse elsewhere. The adaptive mesh refinement technique is extremely useful for applications. It allows not only to consider very complicated geometries but also to treat with appropriate accuracy moving interfaces involving discontinuities, shock waves, phase change and combustion interfaces, or any localized largegradient regions. Among a good many publications which deal with the development and application of the AMR technique books and reviews [1-3] should be mentioned. The AMR approach is also applied successfully to the simulation of flows in porous media [4]. It should

\footnotetext{
${ }^{1}$ Institute for Mathematical Modeling RAS, 4-A Miusskaya Square, Moscow 125047, Russia. E-mail: nata@imamod.ru

${ }^{2}$ Moscow Institute of Physics and Technology (State University), 9, Institutskii per., Dolgoprudny, Moscow Region 141700, Russia.

${ }^{3}$ Moscow State University of Technology "Stankin", 1, Vadkovskij per., Moscow 127994, Russia.
} 
be noted that the AMR technique is not only the subject of theoretical studies but has already been implemented successfully in many in-house as well as commercial CFD codes such as CLAWPACK and AMRCLAW software for conservation laws and hyperbolic systems, Cart3D for inviscid flows, FlowVision, COSMOS FloWorks, CFD ACE+ and CFD FASTRAN for viscous flows at low and high Reynolds number etc. However, an efficient and accurate implementation of this approach faces on serious enough problems and requires a good deal of effort for their resolving.

The present work is aimed at developing efficient adaptive mesh refinement algorithms and applying them to the problem of simulation of multiphase fluid flows in porous media when one of the fluids contains a contaminant. The proposed algorithms permit simultaneous use of different adaptive meshes to predict different physical fields (for the above problem these are the fields of absolute permeability, pressure, water saturation, and suspension concentration). The mesh functions interpolation on going from one to another mesh is implemented in a simple style.

\section{Two-dimensional hierarchical locally refined meshes with dynamic adaptation to the solution}

Let us consider a 2D adaptive mesh consisting of square cells and the algorithms of its functioning. The mesh algorithms and the functionality described in this section are independent of the problems to be solved on the mesh. It provides the ability to implement adaptive mesh algorithms as a self-contained software package.

1.1. Main assumptions. For the formulation of the problem and simplification of the algorithms the following assumptions are introduced.

1. Initially, the mesh consists of a unique rectangular cell $C_{0}$ referred to as the root of the mesh.

2. Each cell $C_{i}(0 \leqslant i<N)$ has size $\left(h_{i}^{x}, h_{i}^{y}\right)$ and stores the value $V_{i}$ (scalar or vector), describing the mean value of the calculated function within the cell area.

3. A cell can be divided (split) into four cells of identical sizes. These smaller cells are referred to as the children of the divided cell. The divided cell itself is called the parent of its children. It is considered that cells which have been split still exist and belong to the mesh. The splitting of the cell means creating its children without deleting the cell itself.

4. Four cells can be merged only if they once made one cell.

5. A cell cannot have cells in its neighborhood which are more than twice smaller than the current cell. The neighborhood of the cell $C_{i}$ is a rectangle measuring $\left(3 h_{i}^{x}, 3 h_{i}^{y}\right)$ whose center coincides with the cell center (see Fig. 1.1). This limitation is stricter than the common limitation where only cells that contact the boundaries or corners should not differ more than twice.

\subsection{Mesh structure.}

Mesh tree. The mesh can be stored in the form of quaternary tree (Fig. 1.2). The leaves of the tree are actual cells on which calculations are made. A leaf cell $C_{i}$ can be split into 4 child cells which will be referred to as $C_{\mathrm{cLU}(i)}, C_{\mathrm{cRU}(i)}, C_{\mathrm{cLB}(i)}, C_{\mathrm{cRB}(i)}$. To maintain the tree structure, each cell $C_{i}$ (in addition to $V_{i}$ ) stores the indexes (or memory pointers) cLU $(i$ ), cRU $(i), \operatorname{cLB}(i), \operatorname{cRB}(i)$ and the index of the parent cell $\mathrm{P}(i)$.

Cell neighbors. It can be assumed that a cell always has exactly 8 neighbors $C_{\mathrm{nLU}(i)}$, $C_{\mathrm{nU}(i)}, C_{\mathrm{nRU}(i)}, C_{\mathrm{nL}(i)}, C_{\mathrm{nR}(i)}, C_{\mathrm{nLB}(i)}, C_{\mathrm{nB}(i)}, C_{\mathrm{nRB}(i)}$ which are not smaller than the current 
cell. These neighbors and their notation are shown in Fig. 1.1 (the neighbors $C_{\mathrm{nR}(i)}$ and $C_{\mathrm{nRB}(i)}$ are in fact the same cell). These neighbors can have children or can be bigger than the current cell. Thus, a cell can have up to 12 "small neighbors" which are children of these 8 "big neighbors". Below the algorithm of finding neighboring cells will be given.

Fig. 1.1. Cell neighborhood. Hatched cells belong to the neighborhood. The situation shown is contrary to assumption 5 because the largest cell has the smallest cells in its neighborhood
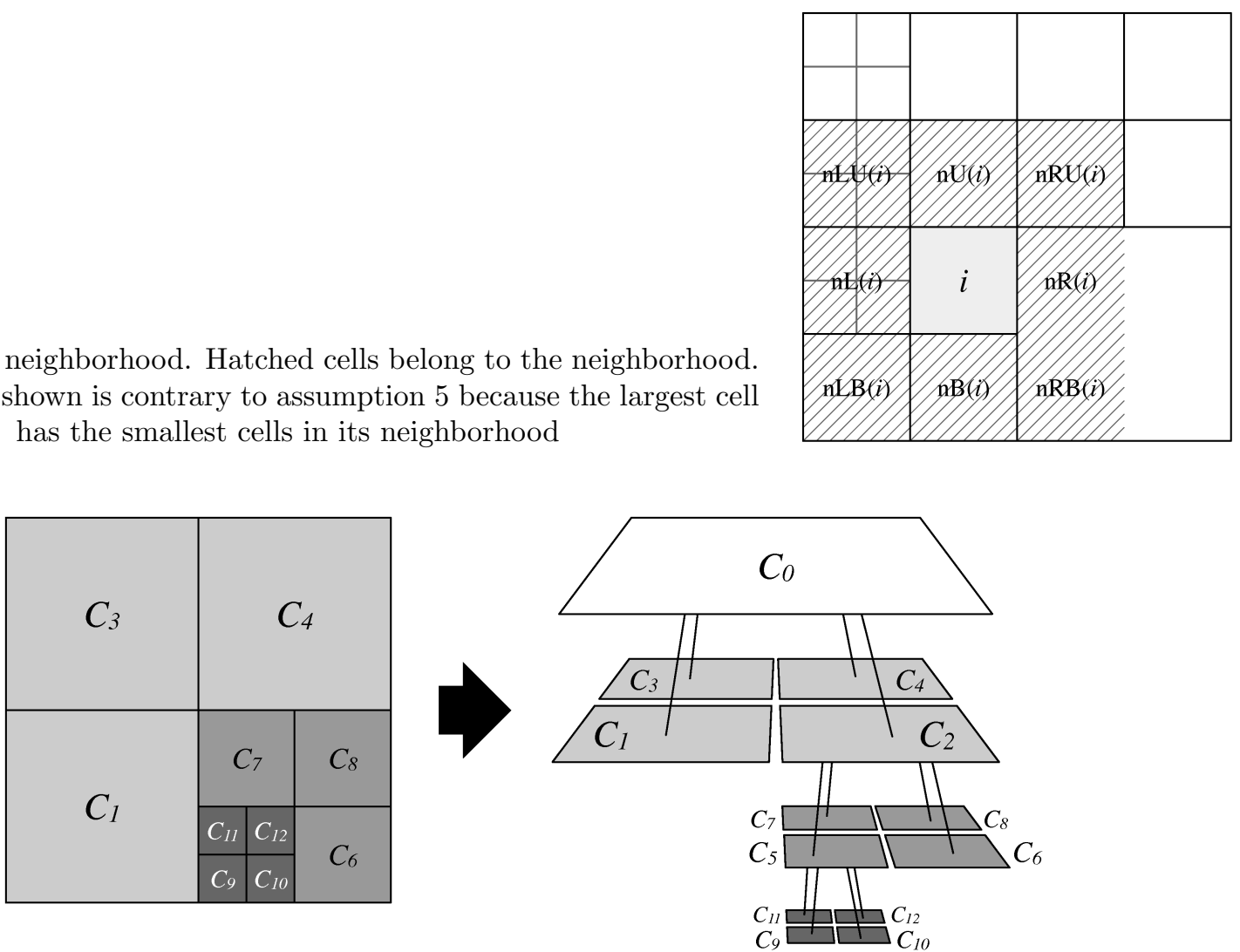

Fig. 1.2. A quaternary tree is a convenient structure for storing a two-dimensional adaptive mesh

Cell address. Besides finding the neighboring cells, one has to determine the cell size and its coordinates. These tasks can be simplified by storing in the cells additional information which will be called the cell address. The cell address $\left(X_{i}, Y_{i}\right)$ is the way from the root cell $C_{0}$ to the cell $C_{i}$. Having the cell address, we can easily find the neighbors' addresses.

Addresses are also used to connect together several adaptive meshes covering the same domain and containing different physical values.

1.3. Interpolation. Let us consider some cell $C$ having the value $V$. When the cell is split into 4 child cells $C_{\mathrm{cLU}}, C_{\mathrm{cRU}}, C_{\mathrm{cLB}}, C_{\mathrm{cRB}}$, they should get such values $V_{\mathrm{cLU}}, V_{\mathrm{cRU}}, V_{\mathrm{cLB}}$, $V_{\mathrm{cRB}}$ that the equality

$$
\frac{V_{\mathrm{cLU}}+V_{\mathrm{cRU}}+V_{\mathrm{cLB}}+V_{\mathrm{cRB}}}{4}=V
$$

takes place. When these cells are merged back, they are destroyed, and their average value is assigned to the parent cell $C$. This is important in order that repeated splitting/merging of the cells does not lead to error accumulation.

However it is not enough to be able to merge and split cells. It is still necessary to solve the following problems:

- it is required to determine values which have to be attributed to child cells upon splitting; 
- it is required to approximate the differential equations on such a mesh;

- it is required to determine which cells have to be agglomerated and which have to be broken down to obtain a sufficiently exact solution using a small enough number of cells (mesh adaptation).

To solve these problems, the interpolation on the mesh has to be built.

Having the values of the mesh cells, we can construct a continuously differentiable interpolation function $f(x, y)$ satisfying the following restrictions for each cell $C$ :

$$
\frac{1}{h^{x} h^{y}} \iint_{C} f(x, y) d x d y=V
$$

Unfortunately, construction of such a function usually requires solving a system of linear equations. Therefore, instead of building the continuously differentiable function $f$, we will build continuous (but not continuously differentiable) functions $\bar{f}, \bar{f}_{x}$ and $\bar{f}_{y}$ which are approximations of $f, f_{x}^{\prime}$ and $f_{y}^{\prime}$ respectively. We will use $\bar{f}_{x}$ and $\bar{f}_{y}$ instead of differentiating the function $\bar{f}$ where necessary.

Let the cell $C$ have neighbors $N_{n_{j}}, j=1, \ldots, 12$ (see Fig. 1.3, some indexes $n_{j}$ may be equal to each other if the cell has less than 12 neighboring cells). It should be split into four square patches $P_{1}, P_{2}, P_{3}, P_{4}$ based on nine points $p_{0}, \ldots, p_{8}$.
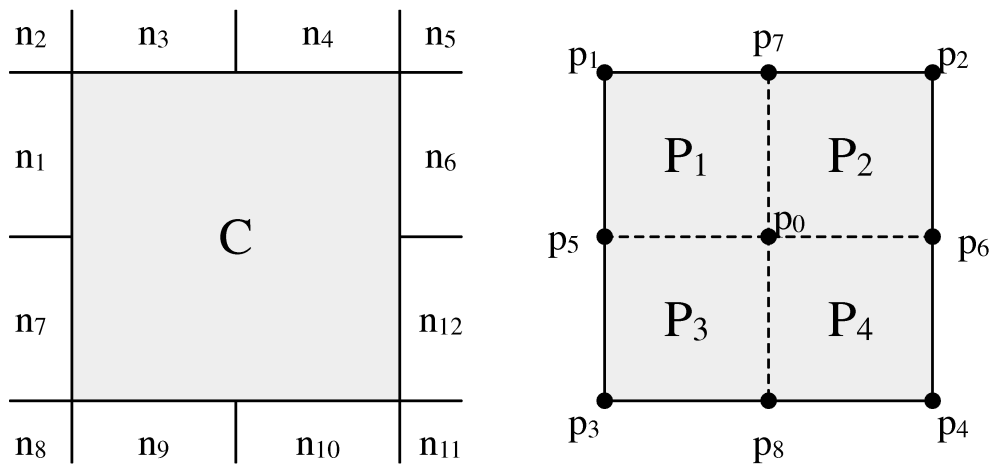

Fig. 1.3. Small neighbors and interpolation points

The approximations $f_{i}, d_{i}^{x}, d_{i}^{y}(i=0, \ldots, 8)$ of the unknown values $f\left(p_{i}\right), f_{x}^{\prime}\left(p_{i}\right)$ and $f_{y}^{\prime}\left(p_{i}\right)$ respectively will be constructed based on these nine points. On each patch the values of $f_{i}, d_{i}^{x}, d_{i}^{y}$ will be interpolated by the bilinear interpolation forming the functions $\bar{f}(x, y), \bar{f}_{x}(x, y)$ and $\bar{f}_{y}(x, y)$.

For each patch $P_{j}, j=1, \ldots, 4$, having corners $p_{i_{1}(j)}, p_{i_{2}(j)}, p_{i_{3}(j)}, p_{i_{4}(j)}$, we require approximations $f_{i_{1}(j)}, f_{i_{2}(j)}, f_{i_{3}(j)}, f_{i_{4}(j)}$ satisfying the following equalities:

$$
\frac{4}{h^{x} h^{y}} \iint_{P_{j}} f(x, y) d x d y=\frac{f_{i_{1}(j)}+f_{i_{2}(j)}+f_{i_{3}(j)}+f_{i_{4}(j)}}{4} .
$$

The combination of equalities (1.3) for $j=1, \ldots, 4$, follows to:

$$
\frac{1}{h^{x} h^{y}} \iint_{C} f(x, y) d x d y \stackrel{(1)}{=} V=\frac{4 f_{0}+f_{1}+f_{2}+f_{3}+f_{4}+2\left(f_{5}+f_{6}+f_{7}+f_{8}\right)}{16} .
$$


It can also be written for the cell edges:

$$
\begin{aligned}
& \frac{1}{h^{y}} \int_{y_{1}}^{y_{2}} f\left(x_{1}, y\right) d y=\frac{2 f_{5}+f_{1}+f_{3}}{4}, \quad \frac{1}{h^{y}} \int_{y_{1}}^{y_{2}} f\left(x_{2}, y\right) d y=\frac{2 f_{6}+f_{2}+f_{4}}{4}, \\
& \frac{1}{h^{x}} \int_{x_{1}}^{x_{2}} f\left(x, y_{1}\right) d x=\frac{2 f_{8}+f_{3}+f_{4}}{4}, \quad \frac{1}{h^{x}} \int_{x_{1}}^{x_{2}} f\left(x, y_{2}\right) d x=\frac{2 f_{7}+f_{1}+f_{2}}{4} .
\end{aligned}
$$

By analogy with (1.4), it will be required for both approximations $d_{i}^{x}$ and $d_{i}^{y}$ that

$$
\begin{gathered}
\frac{1}{h^{x} h^{y}} \iint_{C} f_{x}^{\prime}(x, y) d x d y \stackrel{(4)}{=} \frac{1}{h^{x}}\left(\frac{2 f_{6}+f_{2}+f_{4}}{4}-\frac{2 f_{5}+f_{1}+f_{3}}{4}\right)= \\
\frac{4 d_{0}^{x}+d_{1}^{x}+\ldots+d_{4}^{x}+2\left(d_{5}^{x}+\ldots+d_{8}^{x}\right)}{16} \\
\frac{1}{h^{x} h^{y}} \iint_{C} f_{y}^{\prime}(x, y) d x d y \stackrel{(5)}{=} \frac{1}{h^{y}}\left(\frac{2 f_{7}+f_{1}+f_{2}}{4}-\frac{2 f_{8}+f_{3}+f_{4}}{4}\right)= \\
\frac{4 d_{0}^{y}+d_{1}^{y}+\ldots+d_{4}^{y}+2\left(d_{5}^{y}+\ldots+d_{8}^{y}\right)}{16} .
\end{gathered}
$$

For corner points $(i=1, \ldots, 4)$ the values of $f_{i}, d_{i}^{x}, d_{i}^{y}$ will be found by neglecting the second-order terms in the Taylor series of $f(x, y)$ near these points and assuming that the resulting linear function satisfies equations (1.2) for all cells around the point. In the case where the corner point has 4 adjacent cells, one will get an overdetermined system of equations. It can be solved analytically by the least square technique. The values at the centers of edges $(i=5, \ldots, 8)$ can be found depending on the neighbors sizes (see Fig. 1.4).
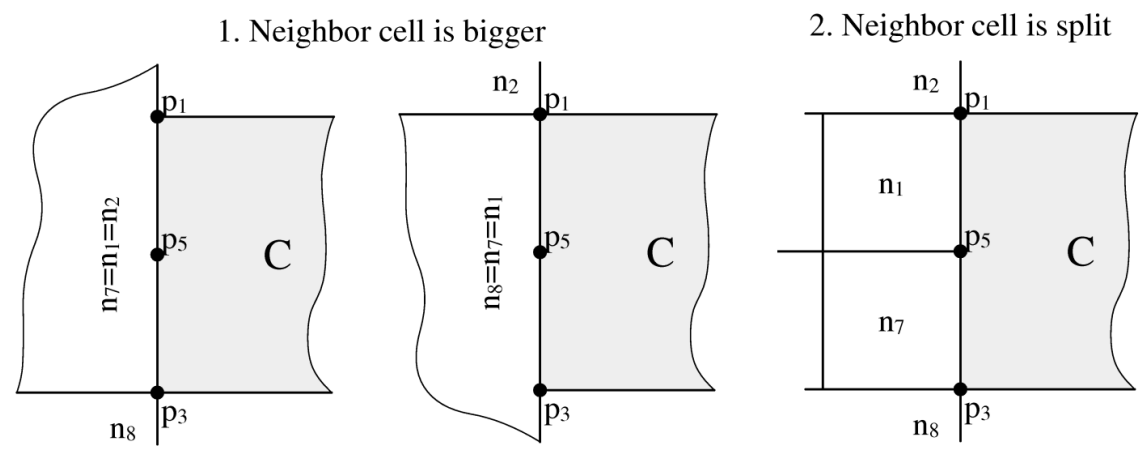

3. Neighbor cell has the same size

Fig. 1.4. Possible neighbor positions near the edge interpolation point

1. If the adjacent neighbor cell is bigger than $C$, then to make the interpolation function continuous, the values at the center of the edge are computed as the mean values of the corresponding corner points $\left(f_{5}=\left(f_{1}+f_{3}\right) / 2, d_{5}^{x}=\left(d_{1}^{x}+d_{3}^{x}\right) / 2, d_{5}^{y}=\left(d_{1}^{y}+d_{3}^{y}\right) / 2\right.$ for the left edge).

2. If the corresponding neighbor cells are smaller than $C$, then the values of the edge interpolation point are computed in the same way as the values at corner points, because the edge point in this case is the corner point for the small neighbors. 
3. If a neighbor has the same size as the current cell, then $f_{i}$ is determined from equations (1.5), (1.6), where the average integral value of the edge is assumed to be equal to the average value of the adjacent cells. The derivative across the edge is assumed to be equal to the difference of adjacent cells values divided by the cell size. The derivative along the edge is calculated as the difference of the corresponding corner values divided by the edge length.

The functions $\bar{f}, \bar{f}_{x}$ and $\bar{f}_{y}$ built at the calculated points by bilinear interpolation give an approximation of $f$ with second order of accuracy and an approximation of $f_{x}^{\prime}$ and $f_{y}^{\prime}$ with first order of accuracy. Nevertheless, the integral values of $\bar{f}_{x}$ and $\bar{f}_{y}$ over the patches (and over the entire cell) approximates the integral values of $f_{x}^{\prime}$ and $f_{y}^{\prime}$ correspondingly with the second order of accuracy.

Having $f_{i}$ one can calculate the values for the new cells $C_{\mathrm{cLU}}, C_{\mathrm{cRU}}, C_{\mathrm{cLB}}, C_{\mathrm{cRB}}$ when splitting the cell $C$

$$
\begin{array}{ll}
V_{\mathrm{cLU}}=\frac{f_{1}+f_{7}+f_{5}+f_{0}}{4}, & V_{\mathrm{cRU}}=\frac{f_{7}+f_{2}+f_{0}+f_{6}}{4}, \\
V_{\mathrm{cLB}}=\frac{f_{5}+f_{0}+f_{3}+f_{8}}{4}, & V_{\mathrm{cRB}}=\frac{f_{0}+f_{6}+f_{8}+f_{4}}{4} .
\end{array}
$$

These values satisfy equality (1.1).

One of the features of the interpolation built is that the functions $\bar{f}_{x}$ and $\bar{f}_{y}$ are not contrary to $\bar{f}$ in the large areas (whose sizes exceed the cells sizes)

$$
\begin{aligned}
& \int_{Y_{1}}^{Y_{2}} \int_{X_{1}}^{X_{2}} \bar{f}_{x}^{\prime}(x, y) d x d y \cong \int_{Y_{1}}^{Y_{2}} \bar{f}\left(X_{2}, y\right) d y-\int_{Y_{1}}^{Y_{2}} \bar{f}\left(X_{1}, y\right) d y, \\
& \int_{Y_{1}}^{Y_{2}} \int_{X_{1}}^{X_{2}} \bar{f}_{y}^{\prime}(x, y) d x d y \cong \int_{X_{1}}^{X_{2}} \bar{f}\left(x, Y_{2}\right) d x-\int_{X_{1}}^{X_{2}} \bar{f}\left(x, Y_{1}\right) d y .
\end{aligned}
$$

Moreover, when the integration borders $X_{1}, X_{2}, Y_{1}, Y_{2}$ coincide with cell borders, the approximate equality (1.10) becomes the exact equality (due to (1.7), (1.8)).

1.4. Mesh function integration. One of the tasks occurring in the building of difference schemes is the calculation of mesh function integrals which can be one- or two-dimensional:

$$
\int_{A}^{B} F\left(\bar{f}, \bar{f}_{x}, \bar{f}_{y}\right) d l, \quad \int_{Y_{1}}^{Y_{2}} \int_{X_{1}}^{X_{2}} G\left(\bar{f}, \bar{f}_{x}, \bar{f}_{y}\right) d x d y
$$

where $A, B,\left(X_{1}, Y_{1}\right),\left(X_{2}, Y_{2}\right)$ are arbitrary points in the mesh area; $F, G$ are some functions (usually non-linear).

The integral over the segment $A B$ can be calculated approximately as follows: the segment $A B$ is divided into small parts by the edges of the interpolation patches (see Fig. 1.5). Then the integral is approximated by the trapezoid method. The two-dimensional integral over the rectangle is approximated in a similar way: each interpolation path intersecting the rectangle $\left(X_{1}, Y_{1}\right),\left(X_{2}, Y_{2}\right)$ forms cell where the integral is approximated by the trapezoid method. 
Cells intersecting $A B$ are sought as follows: the cell containing $A$ (traversing down by the mesh tree) is found, then the integration passes into the neighboring cell according to the edge $A B$ (see Fig. 1.5).

Fig. 1.5. Cells order and points for the approximation of the integral by the trapezoid method

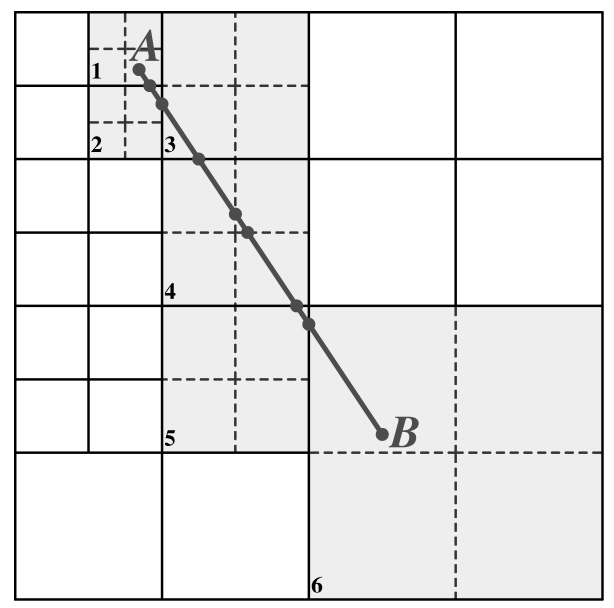

If the problem requires storing several physical value fields, then several adaptive meshes (for example, mesh $M_{1}$ and mesh $M_{2}$ ) are used. Each mesh adapts to its own value field. In this case, we may need to approximate integrals of the following type:

$$
\int_{A}^{B} F\left(\bar{f}^{M_{1}}, \bar{f}_{x}^{M_{1}}, \bar{f}_{y}^{M_{1}}\right) \cdot G\left(\bar{f}^{M_{2}}, \bar{f}_{x}^{M_{2}}, \bar{f}_{y}^{M_{2}}\right) d l .
$$

The points for the trapezoid method in this case are intersections of the segment $A B$ and the interpolation patches of both meshes $M_{1}$ and $M_{2}$. It is important in order to take into account all features presented in both meshes. If one of the functions in (1.11) is linear (for example, $F$ ), then the integral can be calculated implicitly: the value will be the linear combination of $M_{1}$ cells instead of the scalar value.

The following type of integrals will occur when the upwind difference scheme is built (here $A B$ coincides with some of the cell borders):

$$
\int_{A}^{B} F\left(V_{ \pm}^{M_{1}}\right) \cdot G\left(\bar{f}^{M_{2}}, \bar{f}_{x}^{M_{2}}, \bar{f}_{y}^{M_{2}}\right) d l,
$$

Where $V^{M_{1}}$ is the value of the mesh function without interpolation (constant within the leaf cells), $V_{ \pm}^{M_{1}}$ is the $V^{M_{1}}$ value taken at the left or at the right of $A B$ depending on the $G$ value. The integral (1.12) is calculated as follows: first the function $G$ is calculated at the points of intersection of $A B$ and interpolation patches. Then the parts of $A B$ on which $G$ changes the sign are divided by the secants method to find zero points. Between these zero points the integral is approximated by the trapezoid method with respective values of $V^{M_{1}}$.

\subsection{Mesh adaptation.}

Adaptation criterion. To obtain a high accuracy of the solution, the mesh should be adapted to the mesh function that varies on each time step. To perform mesh adaptation some refinement criterion has to be used. As such a criterion one can use the scalar value called the data variation defined by the following formula:

$$
D_{i}=\max _{0 \leqslant j, k \leqslant 8}\left|f_{j}^{i}-f_{k}^{i}\right| \text {. }
$$


In the scalar case (when $f_{j}^{i}$ are scalar values), the data variation for the cell $C_{i}$ is simply the difference between the maximal and the minimal interpolation values over the cell:

$$
D_{i}=\max _{0 \leqslant j \leqslant 8}\left(f_{j}^{i}\right)-\min _{0 \leqslant j \leqslant 8}\left(f_{j}^{i}\right)
$$

Adaptation algorithm. The goal of the mesh adaptation algorithm is to minimize the maximal data variation over the mesh, when the maximum number $N_{\text {need }}$ of leaf cells is fixed:

$$
\max _{i}\left(D_{i}\right) \rightarrow \min , \quad N_{\text {leafs }} \leqslant N_{\text {need }}
$$

where the index $i$ is taken from the set of leaf cells.

Before changing the mesh topology the interpolation function $\bar{f}$ is fixed. After the mesh adaptation is finished the interpolation function is recalculated using the new mesh structure. This is necessary for the following purposes:

1) there is no need to recalculate the interpolation after each splitting/merging while refining;

2) cells values will not depend on the way in which the current mesh structure was obtained from the initial state. This is important to avoid "cyclic" operations during mesh refinement.

The mesh refinement algorithm is described by the flow block shown in Fig. 1.6.

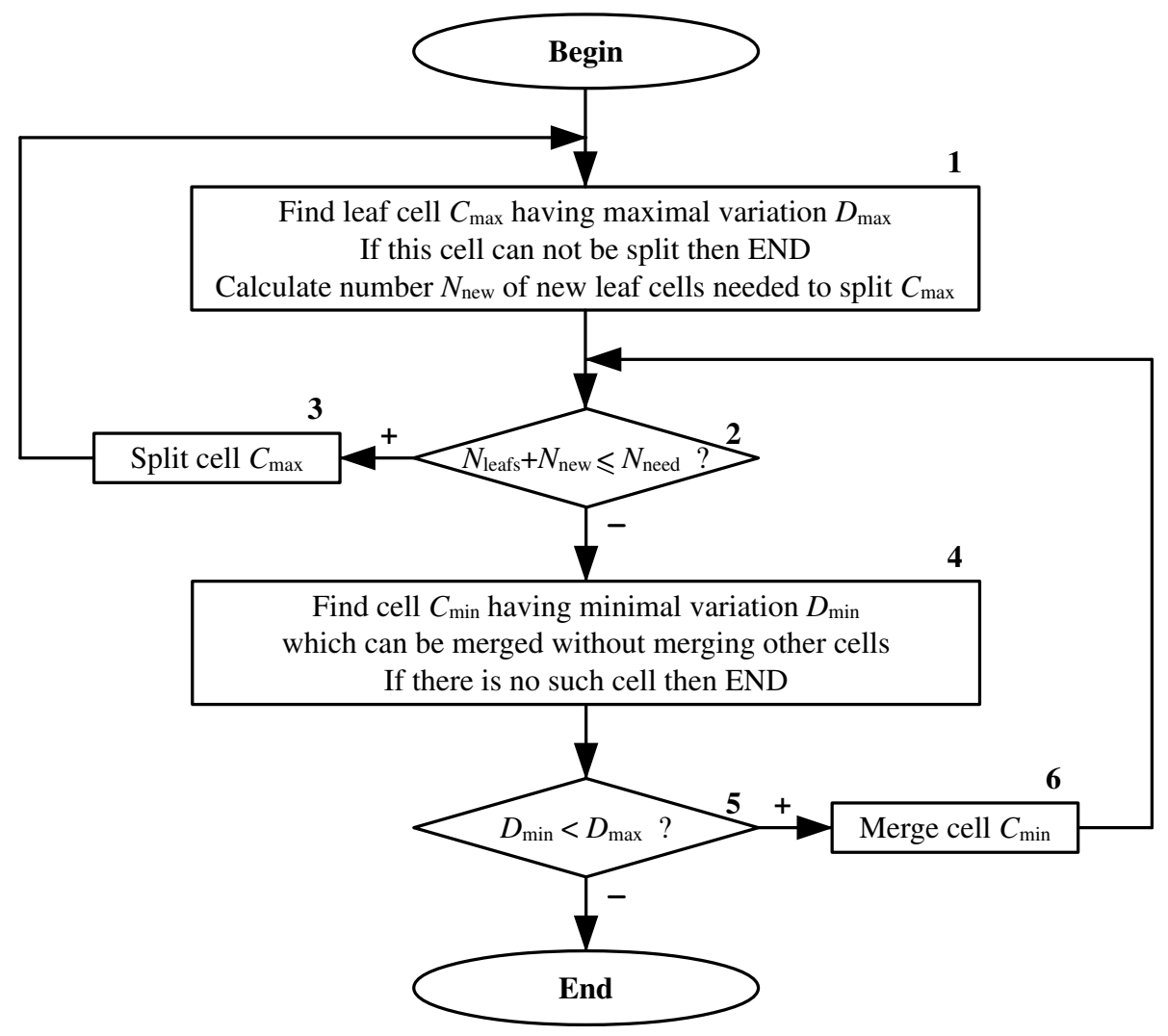

Fig. 1.6. Flow block of the mesh refinement algorithm

1. The only way to reduce $\max \left(D_{i}\right)$ is to split the cell $C_{\max }$ having the maximal variation. To ensure that the adjoining cells do not differ more than twice in size, splitting of the cell can lead to the splitting of neighboring cells. Splitting of neighboring cells can result in the 
splitting of other cells, and so on. This process forms splitting chains. Each splitting in the chain will increase the number of leaf cells by 3. To solve a particular problem, splitting of some cells may be forbidden (for example, to set border conditions). If such a cell gets into the splitting chain then $C_{\max }$ cannot be split. In this case, the refinement algorithm terminates before it reaches the target (1.15).

2 In the condition block 2 , the algorithm checks if there is enough "space" to perform all the splits in the splitting chain.

3. In the program block 3 , the cell $C_{\max }$ is split resulting in the splitting of all of the other cells in the chain. When the cell is split for the first time, its children are positioned in the place of the bilinear interpolation patches of the parent. The successive splitting of the child cells will split these patches, reducing the variation by one half at each splitting.

4. If the splitting requires more leaf cells than specified $\left(N_{\text {need }}\right)$, then one has to merge the cells. The merging procedure consists of deleting all children of some cell (then one can say that this cell is merged). The merged cell can have a higher data variation than its children do, therefore one variation of the cell can become the highest variation in the mesh and the cell will need to be split again. To avoid such a situation, one has to merge the cell having the smallest variation. Unfortunately, merging of a cell can require merging of its neighbors which may have a higher variation. Thus, to ensure that the variation increment due to the merging is minimal, one has to look for the cell $C_{\text {min }}$ with minimal variation $D_{\text {min }}$ among the cells that can be merged without involving the neighbors. The cell $C_{\min }$ should not be a neighbor of the splitting chain to avoid its getting into the chain. If such a cell is absent, the algorithm terminates.

5. In the condition block 5 , the test will be performed if merging will give an advantage and will not lead to an increase in the maximal data variation.

6 . In block 6 , the cell $D_{\text {min }}$ will be merged, decreasing the total number of leaves in the mesh by 3 . This procedure will not affect the cell $C_{\max }$ and its splitting chain. Thus, we do not need to recalculate $C_{\text {max }}$ and $N_{\text {new }}$ and can bypass block 1 .

It has been proved that the algorithm has the following properties if the initial mesh data are not constant throughout the mesh area (if the data are constant, then the mesh can be represented as a single cell) and if there are no restrictions imposed on the splitting and merging of the cells (it can be still restrictions set on the minimal and maximal cell sizes).

1. The refinement algorithm minimizes the maximal data variation in the mesh (it reaches the goal (1.15)).

2. Among all meshes that satisfy (1.15) a structure with the minimal number of cells is found (this structure is unique). This is important for reducing the memory consumption and speeding up computations on such a mesh.

1.6. Parallel realization and load balancing. Parallel computations are very important in the cases where the solution of a large-scale problem is to be obtained in a reasonable time, or where the mesh cannot fit into the memory of a single computer.

The problem to be solved on the adaptive mesh can be parallelized by the domain decomposition technique. The computational domain is split into square areas called "clusters". The clusters are dynamically distributed among the processors as unbreakable units to obtain load balancing and data exchange minimization. Each cluster is covered by an adaptive mesh. These meshes are interconnected providing the way to find the cell neighbors located in the other clusters. Clusters can contain a different number of cells. The event-based MPI mechanism allows simple realization of the program accessing arbitrary cells from neighboring clusters. 
To achieve load balancing, each cluster should be assigned to a processor according to the following criterion:

$$
\max _{i=1, \ldots, n}\left(N_{i}+\alpha_{1} \cdot L_{i}\right)+\alpha_{2} \cdot \max _{i=1, \ldots, n} D_{i} \rightarrow \min .
$$

Here $n$ is the processor number; $N_{i}$ is the number of cells on the $i$-th processor; $L_{i}$ is the total length of the clusters interface between the $i$-th processor and the other processors (measured in cells); $D_{i}$ is the number of cells to be transferred to/from the $i$-th processor to achieve the necessary cluster distribution at the next time step (load balancing overhead expenses).

$\alpha_{1}$ can be estimated precisely enough, knowing the algorithm, the rate of data exchange and the latency. Unfortunately, $\alpha_{2}$ cannot be estimated in that way, because we do not know the number of time steps the clusters will belong to the same processors; we cannot predict the distribution of cells between the clusters until we calculate this distribution. Thus, $\alpha_{2}$ should be estimated by a series of numerical experiments for the given problem.

In the parallel case, each time step consists of the following parts: mesh data calculation, mesh adaptation, and redistribution of clusters.

Assuming that the mesh function changes slowly, we can use the approximate algorithm which continuously modifies the cluster distribution, reducing the value of (1.16) to the local minimum. An important requirement for this method is correct (optimal) initial cluster distribution.

A double-step parallel refinement algorithm has been developed: first the meshes in each cluster are refined independently (preserving the number of cells in each cluster), then all clusters are refined as one big mesh. The algorithm gives an optimal result, but it is not the fastest one.

\section{Passive contaminant transport in an oil-bearing stratum}

At the present time the exploitation of most oil and gas fields is at the closing stage and secondary methods are frequently used, in particular, water flooding. Numerical simulation of these technological methods is usually aimed at developing an optimal strategy of oil reservoir exploitation [5].

As a typical example, the problem of passive contaminant transport in an oil-bearing stratum at water flooding is considered.

2.1. Main assumptions. A joint flow of oil and water in an oil field is considered when oil is extracted by means of non-piston water displacement. It is supposed that the oil field is covered by a network of water injection and oil production wells and the contaminant arrives with water through a number of wells (for example, salty water is injected at some sources).

An oil-bearing stratum is assumed to be thin so that the $2 \mathrm{D}$ problem is considered. The schemes of wells disposition may be quite different. Reference lengths in the problem are hundreds and thousands of meters and reference times are months and years. In these conditions the ratio of the capillary pressure to the full hydrodynamic pressure drop is small. It allows to omit capillary forces so that the flow of two phases w (water) and o (oil) in a porous medium is governed by the classical Buckley-Leverett model [6] based on a number of assumptions: the fluids are immiscible and incompressible, the medium is undeformable, the phase flows comply with the Darcy law, the capillary and gravity forces are neglected. The porosity in the domain is assumed to be a constant value; the permeability is heterogeneous. 
2.2. Equations for the flow in porous media. The Buckley - Leverett model of two-phase flow in porous media is described by the following equations:

$$
\begin{gathered}
m \frac{\partial S}{\partial t}+\operatorname{div}(F(S) K(S) \operatorname{grad} P)=Q^{w}=\left\{\begin{array}{l}
q \cdot F\left(S^{*}\right) \text { - at sources; } \\
q \cdot F(S) \text { - in the whole domain; }
\end{array}\right. \\
\operatorname{div}(K(S) \operatorname{grad} P)=q .
\end{gathered}
$$

Here $m$ is the porosity; $S$ is the water saturation; $F(S)$ is the the Buckley - Leverett function; $K(S)$ is the nonlinear factor including the permeabilities and viscosities; $P$ is the pressure; $q$ denotes the debits of wells; $S^{*}$ is the critical water saturation; the fluid velocity $\mathbf{W}_{f}=K(S) \operatorname{grad} P$ (Darcy law); the water flow velocity $\mathbf{W}_{w}=F(S) \mathbf{W}_{f}$. The nonlinear factors are

$$
F(S)=\frac{k_{w}(S) / \mu_{w}}{k_{w}(S) / \mu_{w}+k_{o}(S) / \mu_{o}}, \quad K(S)=-k \cdot\left(\frac{k_{w}(S)}{\mu_{w}}+\frac{k_{o}(S)}{\mu_{o}}\right) .
$$

Here $k_{w}(S)$ is the relative permeability of water; $k_{o}(S)$ is the oil relative permeability; $k$ is the absolute permeability; $\mu_{w}$ is the water viscosity; $\mu_{o}$ is the oil viscosity.

The relative permeabilities are defined by the following relations $(\underset{\sim}{S}=0.1$ is the residual saturation, $\tilde{S}=0.8$ is the critical saturation, $S_{1}=0.70324, n_{1}=2, n_{2}=2, n_{3}=0.5$ in our tests):

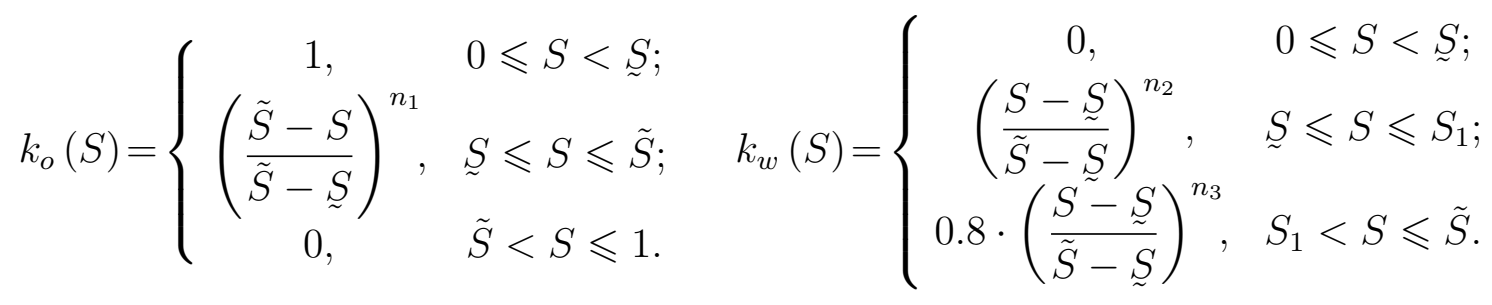

2.3. Equations for passive contaminant transport. To take into account the contaminant transport, the above model should be extended [7]. Suppose that a passive contaminant enters a stratum with water. As the phases are immiscible, the passive contaminant can have a nonzero concentration only in water. Adsorption, convection and diffusion have dominant influence on the contaminant spreading in porous media. The equation for the contaminant concentration can be written as follows:

$$
m \frac{\partial(S \cdot C+a)}{\partial t}+\operatorname{div}\left(\mathbf{W}_{w} C+\mathbf{S}\left(\mathbf{W}_{w}\right)\right)=Q^{c}=Q^{w} \cdot C^{*} .
$$

Here $C$ is the contaminant concentration in water, $a$ is the concentration of the contaminant being adsorbed in a unit volume of a porous medium $(a=a(C)$ for the reversible adsorption), $Q_{C}$ is the source of the contaminant, $C^{*}$ is the contaminant concentration at water sources, $\mathbf{S}$ is the diffusion flow caused by the convective diffusion:

$$
S_{i}=-D_{i j} \frac{\partial C}{\partial x_{j}}, \quad i, j \in\{x, y\} .
$$

$D_{i j}$ is the effective tensor of the convective diffusion. There are phenomenological formulas to obtain it via the Darcy velocity and some characteristics of the medium. In our computations, we use the formula of V. N. Nikolaevskij [8]

$$
D_{i j}=\left[\left(\lambda_{1}-\lambda_{2}\right) \delta_{i j}+\lambda_{2} n_{i} n_{j}\right] W_{w},
$$


where $W_{w}=\left|\mathbf{W}_{w}\right|, \mathbf{n}=\mathbf{W}_{w} / W_{w}, \lambda_{1}$ and $\lambda_{2}$ are some positive factors. One can see that even in an isotropic medium there is a preferential direction caused by the Darcy velocity vector.

2.4. Application of the adaptive meshes. The problem (2.1), (2.2), (2.4) will be solved using the adaptive meshes. Suppose that the porosity is constant and the permeability field is heterogeneous. Four different adaptive meshes will be used: for storing the values of $S_{w}, P, C$ and $1 / k$. The mesh for $1 / k$ will not change with time and is used for storing and integrating the $1 / k$ values. The adsorption $a(C)$ is omitted to simplify the model.

The following implicit scheme is used to approximate equation (2.2):

$$
\frac{1}{h_{i}^{x}}\left(\int_{p_{4}^{i}}^{p_{2}^{i}} K(\bar{S}) \cdot \bar{P}_{x} d y-\int_{p_{3}^{i}}^{p_{1}^{i}} K(\bar{S}) \cdot \bar{P}_{x} d y\right)+\frac{1}{h_{i}^{y}}\left(\int_{p_{1}^{i}}^{p_{2}^{i}} K(\bar{S}) \cdot \bar{P}_{y} d x-\int_{p_{3}^{i}}^{p_{4}^{i}} K(\bar{S}) \cdot \bar{P}_{y} d x\right)=q_{i}
$$

where the integrals are calculated implicitly with respect to the $\bar{P}$ values (see (1.11)), producing a system of linear equations.

Equation (2.1) is approximated by the upwind scheme:

$$
\begin{gathered}
m \frac{\bar{S}_{i}^{n+1}-\bar{S}_{i}^{n}}{\Delta t}+\frac{1}{h_{i}^{x}}\left(\int_{p_{4}^{i}}^{p_{2}^{i}} F_{w}\left(\bar{S}_{ \pm}^{n}\right) \cdot\left(K\left(\bar{S}^{n}\right) \cdot \bar{P}_{x}\right) d y-\int_{p_{3}^{i}}^{p_{1}^{i}} F_{w}\left(\bar{S}_{ \pm}^{n}\right) \cdot\left(K\left(\bar{S}^{n}\right) \cdot \bar{P}_{x}\right) d y\right)+ \\
\frac{1}{h_{i}^{y}}\left(\int_{p_{1}^{i}}^{p_{2}^{i}} F_{w}\left(\bar{S}_{ \pm}^{n}\right) \cdot\left(K\left(\bar{S}^{n}\right) \cdot \bar{P}_{y}\right) d x-\int_{p_{3}^{i}}^{p_{4}^{i}} F_{w}\left(\bar{S}_{ \pm}^{n}\right) \cdot\left(K\left(\bar{S}^{n}\right) \cdot \bar{P}_{y}\right) d x\right)=Q_{i}^{w},
\end{gathered}
$$

where the integrals are approximated by the upwind method (see (1.12)).

Having the pressure field and the water saturation field (at the current and the next time steps) equation (2.4) can be approximated as follows:

$$
\begin{gathered}
m \frac{\bar{C}_{i}^{n+1} \cdot \bar{S}_{i}^{n+1}-\bar{C}_{i}^{n} \cdot \bar{S}_{i}^{n}}{\Delta t}+ \\
\frac{1}{h_{i}^{x}}\left(\int_{p_{4}^{i}}^{p_{2}^{i}} \bar{C} \cdot W_{w}^{x}-D_{x x} \cdot \bar{C}_{x}-D_{x y} \cdot \bar{C}_{y} d y-\int_{p_{3}^{i}}^{p_{1}^{i}} \bar{C} \cdot W_{w}^{x}-D_{x x} \cdot \bar{C}_{x}-D_{x y} \cdot \bar{C}_{y} d y\right)+ \\
\left.\frac{1}{h_{i}^{y}} \underset{p_{1}^{i}}{p_{2}^{i}} \bar{C} \cdot W_{w}^{y}-D_{y x} \cdot \bar{C}_{x}-D_{y y} \cdot \bar{C}_{y} d x-\int_{p_{3}^{i}}^{p_{i}} \bar{C} \cdot W_{w}^{y}-D_{y x} \cdot \bar{C}_{x}-D_{y y} \cdot \bar{C}_{y} d x\right)=Q_{i}^{c},
\end{gathered}
$$

where $W_{w}^{x}=F_{w}\left(\bar{S}^{n}\right) \cdot K\left(\bar{S}^{n}\right) \cdot \bar{P}_{x}, W_{w}^{y}=F_{w}\left(\bar{S}^{n}\right) \cdot K\left(\bar{S}^{n}\right) \cdot \bar{P}_{y}$. It is not necessary to use the upwind scheme in this case, because the diffusion flow $\mathbf{S}$ will provide monotonous results.

2.5. Results of numerical experiments. First of all the tools developed for adaptive mesh refinement were approved by solving a convection problem with a known exact solution [9]. Comparison of the results obtained on uniform and on adaptive meshes demonstrated the following time advantage of the adaptive meshes: the run time on the adaptive mesh 
was 1.5 times longer than on a uniform mesh with the same number of cells, but to achieve the same precision on the uniform mesh 16 times more cells were needed. Thus, having the same precision, the solution of the given test problem could be obtained about 10 times faster with the use of the adaptive mesh.

The 5 spot-pattern problem will be investigated as a test. The physical domain is square $(150 \times 150$ meters $)$. The injection well is in the left bottom corner, the productive well is in the right top corner. The permeability field is shown in Fig. 2.1. The absolute permeability varies from $10^{-8}$ to $10^{-12} \mathrm{~m}^{2}$. The other model parameters are $m=0.2$, the well debit is $300 \mathrm{~m}^{3} /$ day, the height of the oil layer is 10 meters (it is necessary to translate the debit to the $2 \mathrm{D}$ problem formulation), $\mu_{w}=1.2 \cdot 10^{-13}, \mu_{o}=6 \cdot 10^{-13}$. The results of the simulation (100 days of oil recovery) are shown in Fig. 2.2. The saturation maximum, the concentration, and the pressure correspond to the water injection well (left bottom corner) that supplies the stratum with the contaminant. The oil production well is in the right top corner. The moving fronts have complex shapes and one can see the so-called "fingering".

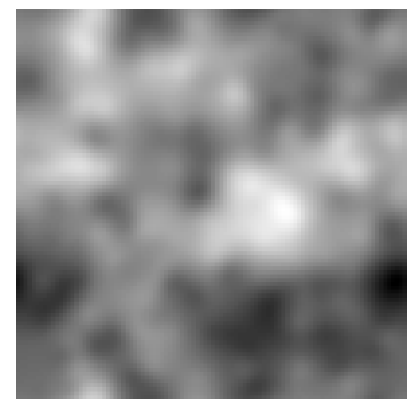

Fig. 2.1. The permeability field. Black color: $k=10^{-12} \mathrm{~m}^{2}$, white: $k=10^{-8} \mathrm{~m}^{2}$

The calculations were performed on the self-made cluster consisting of 14 two-processor two-kernel modules with a peak performance of 300 Flops. The developed parallel mesh refinement tools permit determining the optimal number of processors for the given quantity of mesh cells from the standpoint of load balancing. For example, for a coarse enough mesh consisting of 4096 cells the optimal number of processors is 3. Fig. 2.3 shows the mesh distribution among 4 processors. The fourth processor (red) is most of the time completely switched off. This is due to the large amount of data exchange compared to the calculation time (13300 cells in total in 4 meshes).

\section{Conclusions}

New algorithms of 2D adaptive mesh refinement have been constructed and validated on the problem of passive contaminant transport in an oil-bearing stratum at water flooding in a heterogeneous medium. The method of adaptive meshes significantly reduces the computational time and memory requirements compared to the uniform meshes having a similar accuracy of solution. A parallel implementation of the developed algorithms with dynamic load balancing has been constructed.

The adaptive meshes will find wide application as a software package.

Acknowledgements. The work was supported by the INTAS Ref. No. 03-50-4395 and by the Russian Foundation for Basic Research, (Grants 05-01-00510, 06-01-00187). 

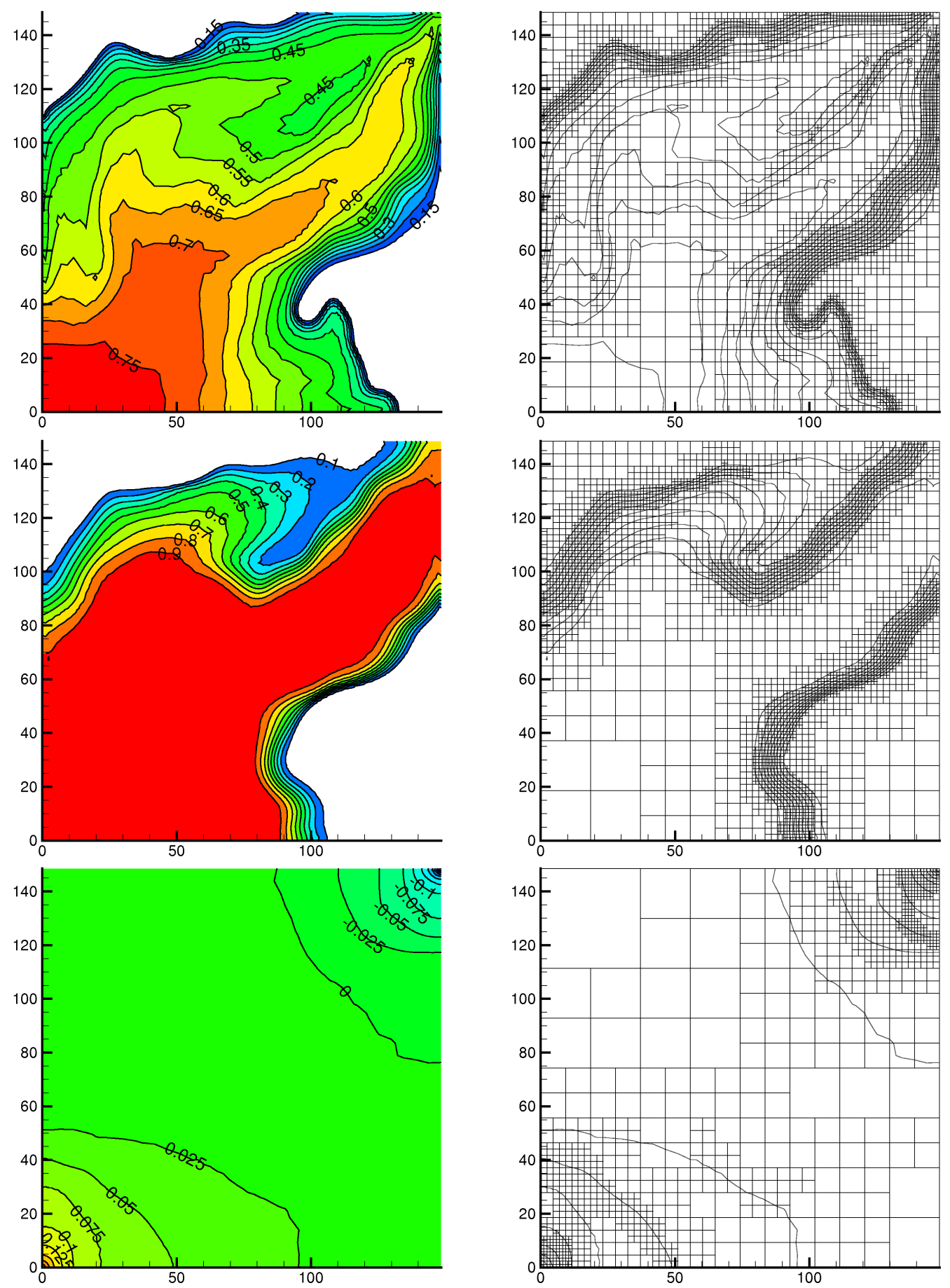

Fig.2.2. Computed physical fields and their corresponding meshes: water saturation (top, 4096 cells), suspension concentration (midst, 4096 cells), and pressure (bottom, 1024 cells) 

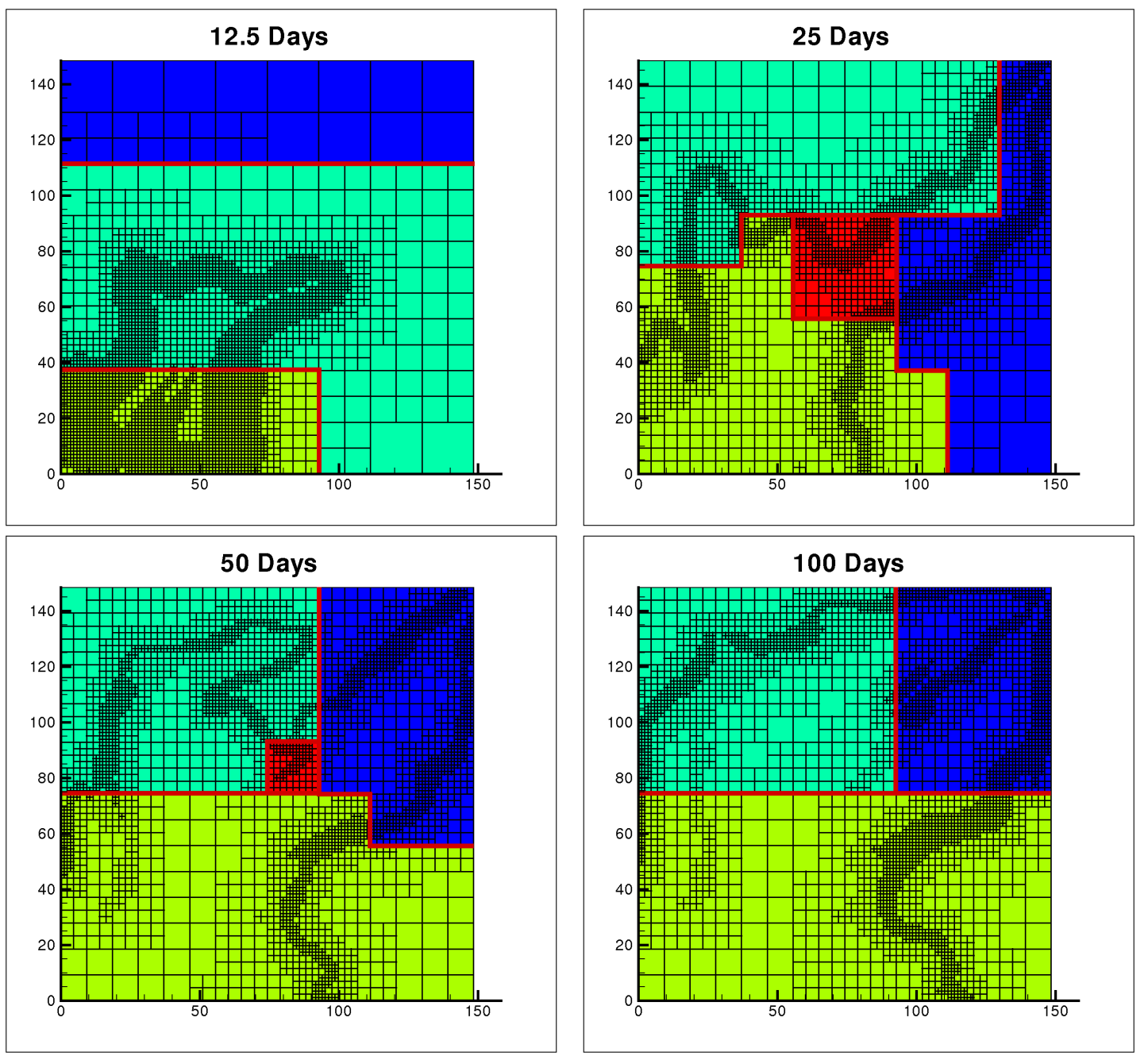

Fig.2.3. Distribution of the mesh among the processors. The colors show the processor number

\section{References}

1. P. J. Frey and P.-L. George, Mesh Generation. Application to Finite Elements, HERMES Science Publishing, Oxford, UK, 2000.

2. V. D. Liseikin, Grid Generation Methods, Springer-Verlag, Berlin, 1999.

3. M. J. Aftosmis Solution adaptive cartesian grid methods for aerodynamic flows with complex geometries, in: Lecture Notes for 28th CFD Lecture Series, von Karman Institute for Fluid Dynamics, Belgium, 1997, pp. 1-108.

4. J. A. Trangenstein, Multi-scale iterative techniques and adaptive mesh refinement for flow in porous media, Advances in Water Resources, 25 (2002), pp. 1175-1213.

5. M. A. Trapeznikova and N. G. Churbanova, Simulation of multi-phase fluid filtration on parallel computers with distributed memory, in: Proc. of the 4th European CFD Conf. (Eds K. D. Papailiou et al.), Wiley, Chichester, 1998, Vol.1, Pt.2, pp. 929-934.

6. R. E. Ewing (Ed.), The mathematics of reservoir simulations, SIAM, Philadelphia, 1983.

7. V. M. Entov and A. F. Zazovskij, Hydrodynamics of processes for oil production acceleration, Nedra, Moscow, 1989 (in Russian).

8. V. N. Nikolaevskij, Mechanics of porous and fissured media, Nedra, Moscow, 1984 (in Russian).

9. B. N. Chetverushkin, N. G. Churbanova, A. A. Soukhinov, and M. A. Trapeznikova Simulation of contaminant transport in an oil-bearing stratum at water flooding, in: Proc. of ECCOMAS CFD 06 (Eds P. Wesseling, E. Onate, and J. Periux), TU Delft, The Netherlands, 2006. 